\title{
Optical Properties of Novel Azo Multi-Functional Dye Thin Films
}

\author{
Ani Stoilova, Yordanka Trifonova, Vanya Lilova \\ University of Chemical Technology and Metallurgy \\ 8 Kliment Ohridski blvd., Sofia \\ Bulgaria, Department of Physics \\ ani_st@web.de
}

\section{Extended Abstract}

By evaluation the practical applicability of new synthesized compounds, starting point represents the thin film optical properties analysis, which provides rich information about chemical bonding, layer thickness, electronic structure, etc.

Here, we present optical properties determination of thin nanocomposite films of three novel N-phthalimide azoazomethine dyes containing $\mathrm{OH}$-group at ortho-position in respect to the $-\mathrm{CH}=\mathrm{N}$ - chromophore group and designed with different electron donating and electron withdrawing substituents. The structure of these previously synthesized by us dyes is shown below.

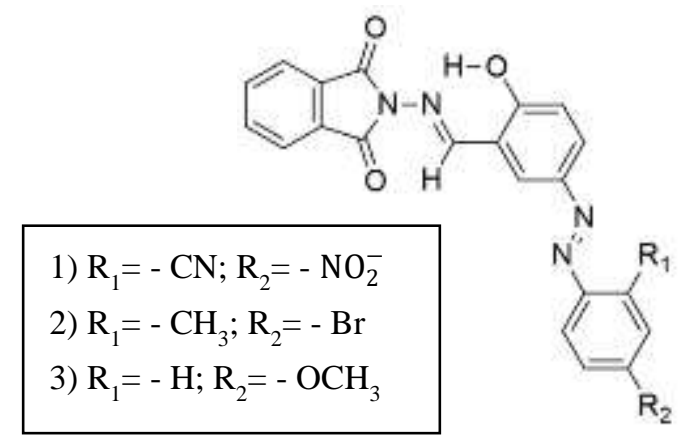

With the aim to explore potential practical application of these novel azo multi-functional dyes, we prepared thin nanocomposite films via vacuum thermal evaporation and low temperature spray technique, and determined the transmittance coefficient $(\mathrm{T})$, the reflectance coefficient $(\mathrm{R})$, the refractive index $(\mathrm{n})$, the extinction coefficient $(\mathrm{k})$, the optical absorption coefficient $(\alpha)$ and the optical band gap $(\mathrm{Eg})$ of the fabricated thin films in the spectral range between 340 and $800 \mathrm{~nm}$. The thin films prepared through vacuum thermal evaporation show higher transmittance, higher reflectance, higher refractive index $(n=1.5 \div 2.1)$ and higher values of the optical band gap $(\mathrm{Eg}=2.93 \div 3.53, \mathrm{eV})$. The effect of the substitutions and of the fabrication method on the optical performance of the nanocomposite thin films has been discussed.

Keywords: Azo dyes, Optical properties, Nanocomposite thin films.

\section{Acknowledgments}

Y. Trifonova and V. Lilova would like to thank the Bulgarian National Scientific Fund, Project ДН 18/5 of the Ministry of Education and Science for supporting this research work.

A. Stoilova thanks the Bulgarian National Scientific Fund, project ДН 08/10 of the Ministry of Education and Science for supporting this research work. 